\title{
Correction: Sodium/potassium ratio change was associated with blood pressure change: possibility of population approach for sodium/potassium ratio reduction in health checkup
}

Mana Kogure - Naoki Nakaya - Takumi Hirata - Naho Tsuchiya - Tomohiro Nakamura • Akira Narita • Yoko Suto • Yoko Honma · Hidemi Sasaki • Ken Miyagawa • Yusuke Ushida · Hiroyuki Ueda • Atsushi Hozawa

Published online: 8 September 2020

(c) The Japanese Society of Hypertension 2020

Correction to: Hypertension Research https://doi.org/10.1038/s41440-020-00536-7 published online 17 August 2020

Conflict of interest should read as follows:

\section{Conflicts of interest}

$\mathrm{KM}$ is an employee of OMRON Healthcare Co., Ltd. YU and HU are employees of Kagome Co., Ltd. The authors declare that they have no conflict of interest. 